Gut, 1973, 14, 204-208

\title{
The effect of coeliac disease upon bile salts
}

\author{
T. S. LOW-BEER, ${ }^{1}$ K. W. HEATON, E. W. POMARE, AND A. E. READ \\ From the University Department of Medicine, Bristol Royal Infirmary
}

SUMMARY The size and composition of the bile salt pool has been measured in patients with untreated coeliac disease and in control subjects. The total bile salt pool was markedly increased in coeliac patients, the average being 9.2 grams compared with 3.1 grams in controls. Taurocholate synthesis was normal, consistent with its enlarged pool and prolonged half-life. Half-life and pool size were significantly correlated. The composition of the bile salt pool was virtually identical in the two groups. Our findings suggest that as the enterohepatic circulation is slowed by gallbladder inertia, so hepatic surveillance of pool size is diminished.

We have recently shown that in patients with untreated coeliac disease the gallbladder is relatively inert and the enterohepatic circulation of bile salts more sluggish than normal (Low-Beer, Heaton, Heaton, and Read, 1971). We attributed this to a failure of the damaged upper small intestinal mucosa to release cholecystokinin. This paper is concerned with the effect of the disease on the size of the bile salt pool, its composition, and its daily rate of turnover.

\section{Subjects and Methods}

Patients with adult coeliac disease, in whom jejunal biopsy showed subtotal villous atrophy, and who were taking an unrestricted diet, volunteered to undertake these studies; they were compared with a group of age-matched volunteers with no history of gastrointestinal disease drawn from members of staff, hospital outpatients, and medical students. All analyses were performed on bile aspirated after an overnight fast through a tube in the duodenum. Bile flow was stimulated by an iv injection of 10-40 Ivy dog units of cholecystokinin.

MEASUREMENT OF THE COMPOSITION OF THE BILE SALTS IN DUODENAL BILE

Measurements were made in 14 patients and 17 control subjects. The molar quantities of the glycine conjugates of the trihydroxy (cholic) and dihydroxy (deoxycholic and chenodeoxycholic) bile salts in

${ }^{1}$ Requests for reprints should be addressed to Dr T. S. Low-Beer, University Department of Medicine, Royal Infirmary, Bristol, BS2 8HW.

Received for publication 19 September 1972. each sample were measured enzymatically (Iwata and Yamasaki, 1964) after thin-layer chromatographic separation (Hofmann, 1962). Deoxycholate was measured directly by a modification of the salicylaldehyde colour reaction and the amount of chenodeoxycholate in the mixture derived by subtraction (Bruusgaard, 1970). The specificity of the salicylaldehyde method for measuring deoxycholic acid is shown in figure 1 . In this experiment, the effect of increasing amounts of chenodeoxycholic acid on the absorbance at $700 \mu$ due to fixed amounts of glycodeoxycholic acid was measured. Chenodeoxycholic acid produced $3 \%$ of the absorbance compared with equimolar amounts of glyco-

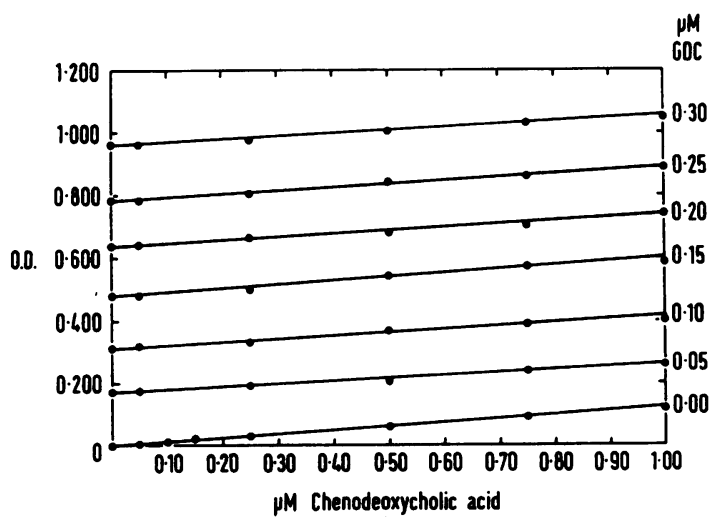

Fig. 1 The specificity of the salicylaldehyde reaction for deoxycholic acid in the presence of its isomer, chenodeoxycholic acid. 
deoxycholic acid. This agrees precisely with Bruusgaard's findings. When a single sample of bile was extracted and analysed on six separate occasions, the following coefficients of variation of the mean were obtained: glycocholate $2 \cdot 1 \%$, glycochenodeoxycholate $4.5 \%$, glycodeoxycholate $3.8 \%$. The glycine to taurine conjugation ratio was calculated by measuring the total glycine and taurine conjugates enzymatically. The coefficient of variation of the mean calculated as above was $1.7 \%$. In most cases bile samples aspirated on several days from the same patient were analysed. The results were found to agree closely with each other.

\section{MEASUREMENT OF TAUROCHOLATE POOL}

\section{SIZE AND TURNOVER AND OF TOTAL BILE}

SALT POOL

Studies were performed using carboxyl-14 C-labelled sodium taurocholate. This material was obtained from Tracerlab (Weybridge, England) and the Radiochemical Centre (Amersham, England). The former had to be purified by preparative thin-layer chromatography to reach the $99 \%$ radiochemical purity of the latter product.

Ten patients and 17 control subjects had a measured amount (approximately $5 \mu \mathrm{Ci}$ of radioactive taurocholate) injected intravenously on the first morning. On four mornings during the subsequent four to seven days duodenal bile was aspirated and 3-5 $\mathrm{ml}$ retained for analysis. The method for separating taurocholic acid by thin-layer chromatography and measuring its rate of fall of specific activity in duodenal contents, and of calculating pool size and daily turnover, has previously been described (Austad, Lack, and Tyor, 1967; Heaton, Austad, Lack, and Tyor, 1968). The correlation coefficient (r), representing the goodness of fit of the regression line (log specific activity versus time), was calculated for each patient. In some of the studies taurocholic acid was estimated using the 3- $\alpha$ hydroxysteroid dehydrogenase enzyme. From a knowledge of the taurocholate pool size and of the proportion of the different bile salts in the samples, the total pool of bile salts was calculated. This was carried out in all the patients and in 11 of the control subjects in whom the composition of the bile salt pool was measured.

\section{Statistical Methods}

Those used are the Wilcoxon and White rank sum test, and linear regression analysis.

\section{Results}

The table shows the proportion of the three main glycine-conjugated bile salts and the ratio of glycine to taurine conjugates in duodenal aspirates from patients with coeliac disease and control subjects. The values are very similar in the two groups and there is clearly no alteration in the composition of the bile salt pool in coeliac disease.

Figure 2 shows a significant enlargement of the taurocholate pool in coeliac patients compared with the control subjects. The average of $1114 \mathrm{mg}$ is three times greater than the $342 \mathrm{mg}$ in the controls. Seven of the 10 coeliac patients have a taurocholate pool larger than any of the 17 control subjects. Since the bile salt composition is similar in the two groups, the total bile salt pool of the coeliac patients is greatly expanded also, as shown in figure 3 . The average total bile salt pool is $9.2 \mathrm{~g}$ in the coeliac patients compared with $3.1 \mathrm{~g}$ in the controls.

When the size of the taurocholate pool is plotted against its half-life in patients with coeliac disease, the two measurements are correlated: as the halflife increases, so does the size of the pool (fig 4) If one excludes from the calculations the patient with an extraordinarily long half-life of 18.4 days, a highly significant regression line can be constructed. Even in the excluded patient, the taurocholate pool is almost five times the size of the average control subject. In the control subjects, no correlation between the taurocholate pool size and its half-life was evident.

The daily turnover of taurocholate, that is, the amount that is excreted and replaced daily by hepatic synthesis, is a function of both pool size and halflife. It is calculated from the formula

$$
\text { turnover }=\frac{\text { pool size } \times 0.693}{\text { half-life }}
$$

(Austad et al, 1967; Hepner, Hofmann, and Thomas, 1972). When the taurocholate turnover is plotted,

\begin{tabular}{|c|c|c|c|c|}
\hline \multirow[t]{2}{*}{ Subject } & \multicolumn{3}{|c|}{ Percentage in Each Fraction (mean $\pm 1 S D$ ) of Glycine-conjugated Bile Salts } & \multirow{2}{*}{$\begin{array}{l}\text { Ratio of Glycine to Taurine Conjugation } \\
(\text { mean } \pm 1 S D)\end{array}$} \\
\hline & Cholate & Chenodeoxycholate & Deoxycholate & \\
\hline $\begin{array}{l}\text { Coeliac disease } \\
(\mathrm{n}=14)\end{array}$ & $40 \cdot 2 \pm 7 \cdot 4$ & $34 \cdot 1 \pm 10 \cdot 4$ & $25 \cdot 8 \pm 11 \cdot 7$ & $3 \cdot 0 \pm 1 \cdot 7$ \\
\hline $\begin{array}{l}\text { Control } \\
(\mathrm{n}=17)\end{array}$ & $36 \cdot 6 \pm 5 \cdot 3$ & $37 \cdot 1 \pm 8 \cdot 5$ & $26 \cdot 3 \pm 8 \cdot 0$ & $2 \cdot 9 \pm 1 \cdot 3$ \\
\hline
\end{tabular}

Table Composition of the bile pool in patients with coeliac disease and control subjects 
POOL SIZE $g$.

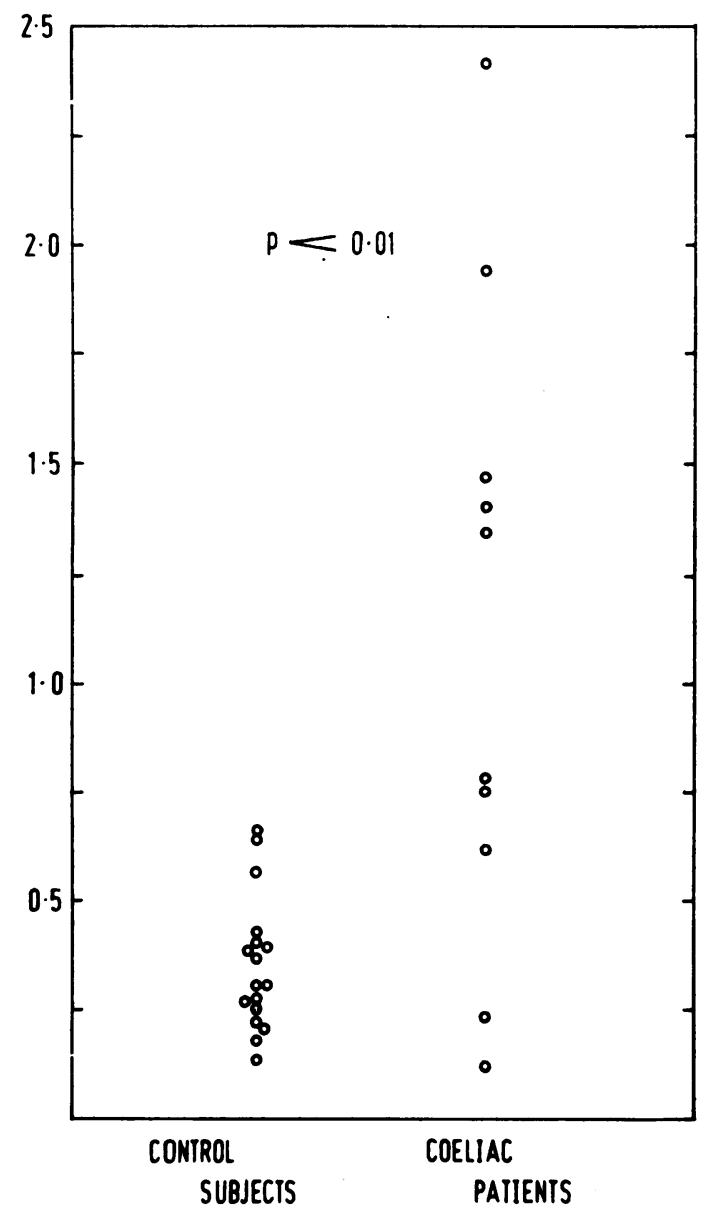

Fig. 2 Taurocholate pool size in coeliac patients and control subjects.

POOL SIZE $g$.

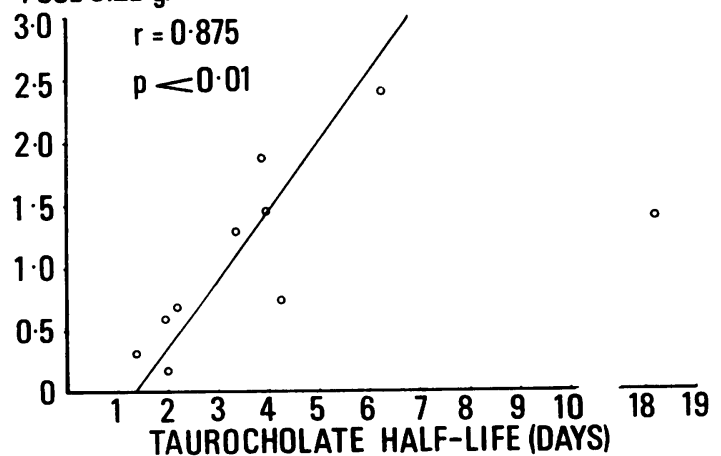

Fig. 4 Taurocholate pool size plotted against taurocholate half-life. The calculation of the regression line (sum of least squares) does not take account of the point at 18.4 days.

$$
\text { POOL SIZE } g \text {. }
$$$$
p=0.01
$$

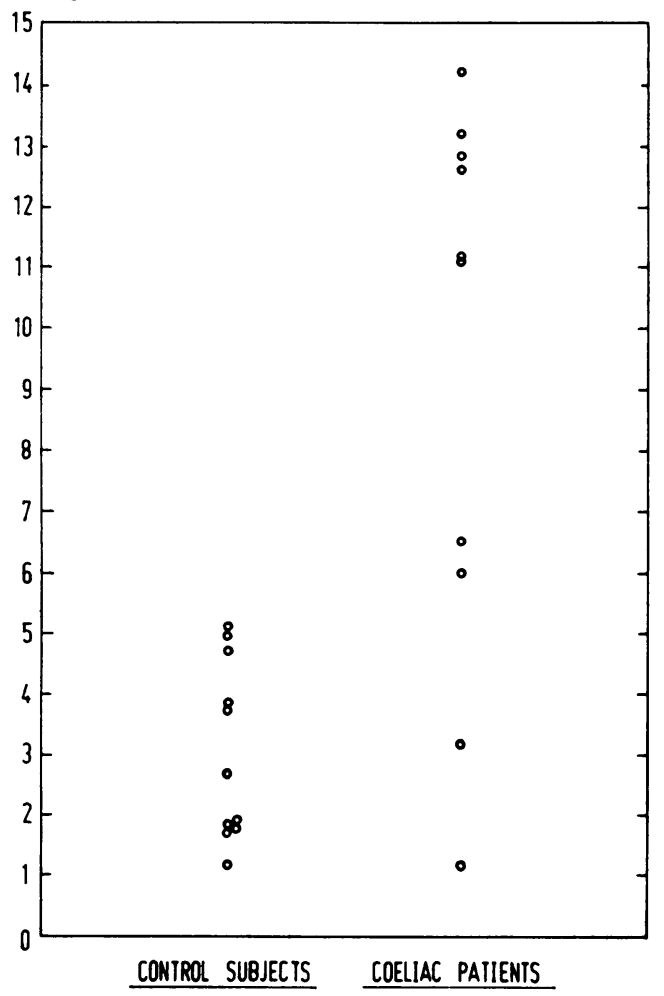

Fig. 3 Total bile salt pool size in coeliac patients and control subjects.

as in fig 5 , the values obtained are not appreciably different in coeliac patients $(195 \pm 102 \mathrm{mg})$ and the controls (182 $\pm 99 \mathrm{mg})$.

\section{Discussion}

This study has shown that patients with coeliac disease have a bile salt pool which is markedly enlarged but normal in composition. Enlargement of the bile salt pool has never previously been described in man.

In a recent paper (Low-Beer et al,1971), we showed that labelled taurocholate is metabolized and excreted more slowly than normal in coeliac disease and that gallbladder contraction in response to a fatty meal is impaired. When interpreting the present findings a number of possibilities have been considered. First, the results are unlikely to be due to inadequate mixing of the isotope with inert gallbladder contents, since this would tend to give spuriously high readings of taurocholate specific activity, especially on the first day, and consequently 


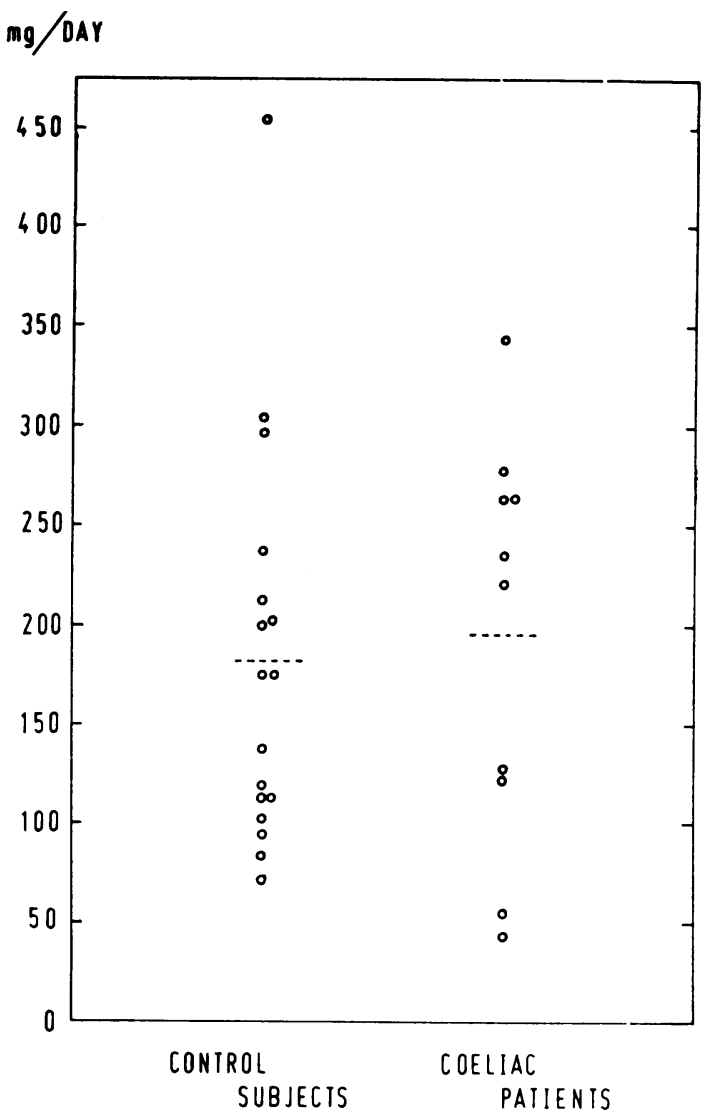

Fig. 5 Daily turnover of taurocholic acid in coeliac patients and control subjects.

a spuriously low value for pool size. However, the pool sizes were large, and we did not find the specific activity on the first day to be higher than on the other days, all the readings falling close to the calculated regression lines (mean ' $r$ ' value $=0.984$, range 0.948-0.997).

Secondly, ileal reabsorption may be more complete than normal due to the characteristically slow transit of small intestinal contents in coeliac disease (Spiro, 1970; Jeans, 1972), or to an increased functional capacity of the ileum, in compensation for jejunal disease (Dowling, 1972). This could account for a reduced rate of turnover and bacterial metabolism since more of the bile salt pool than normal would return to the liver, and less enter the colon. However, increased return to the liver would suppress hepatic synthesis by negative feedback Low-Beer, Pomere, and Morris (1972). This reduction in hepatic synthesis would tend to compensate for the increased return of bile salts and prevent the pool from expanding. Therefore, increased ileal reabsorption, while not excluded, by itself fails to explain the expanded bile salt pool.

Our own hypothesis may be spelt out as follows. The primary abnormality is biliary stasis, caused by lack of cholecystokinin release and hence understimulation of gallbladder contraction and impaired Oddi sphincter relaxation. Biliary stasis, with sequestration of the bile salt pool in the gallbladder, results in less bile entering the intestine. Inevitably this means a reduced return of bile salts to the liver, and reduces feedback inhibition of hepatic bile salt synthesis. The rate of this synthesis therefore increases, although there has been no corresponding increase in the loss of bile salt from the body. The newly synthesized bile salts are added to those still sequestered in the gallbladder and biliary tree and the pool is expanded. This process continues until a new steady state is reached. At this point a decreased recirculation of an increased pool is quantitatively the same as a normal recirculation of a normal pool, that is, a normal daily turnover.

This hypothesis would explain our finding that taurocholate turnover is normal in the coeliac patients. The strong relationship between the taurocholate half-life and pool size is further evidence that as the enterohepatic circulation is slowed by gallbladder inertia, bile salts are less efficiently fed back to the liver and consequently hepatic surveillance of pool size is diminished.

We have found the composition of the bile salt pool to be normal in coeliac patients. In particular there is no change in the proportion of the bacterial metabolite deoxycholate. This suggests that the extent to which circulating bile salts are exposed to intestinal bacteria is not significantly different from normal. Miettinen and Siurala (1971) and Di Magno, Go, and Summerskill (1972) actually showed a decrease in the proportion of deoxycholate in coeliac bile. We cannot account for this discrepancy. Our previous finding (Low-Beer et al, 1971) that radioactive taurocholate is metabolized slowly is explained by gallbladder inertia and reduced entry into the intestine of the labelled taurocholate pool.

In conclusion, we suggest that in coeliac disease the bile salt pool is enlarged due to reduced hepatic surveillance of bile salt pool size. Because of gallbladder inertia, the pool is turned over and metabolized slowly. We consider that there is no reduction in the absolute amount of bile salts turned over, both because of our own findings with taurocholate and because Meittinen found normal or even slightly increased faecal excretion of total bile salts in patients with gluten enteropathy (Miettinen, 1968).

This work leads us to postulate that in coeliac disease the size of the bile salt pool is partly determined by the frequency of its enterohepatic circulation. 
We wish to thank Dr Susan Heaton for expert technical assistance.

\section{References}

Austad, W. I., Lack, L., and Tyor, M. P. (1967). Importance of bile acids and of an intact distal small intestine for fat absorption. Gastroenterology, 52, 638-646.

Bruusgaard, A. (1970). Quantitative determination of the major 3hydroxy bile acids in biological material after thin-layer chromatographic separation. Clin. Chim. Acta (Amst.), 28, 495 504.

DiMagno, E. P., Go, V. L. W., and Summerskill, W. H. J. (1972). Impaired cholecystokinin-pancreozymin secretion, intraluminal dilution, and maldigestion of fat in sprue. Gastroenterology, 63, 25-32.

Dowling, R. H. (1972). Personal communication.

Heaton, K. W., Austad, W. I., Lack, L., and Tyor, M. P. (1968). Enterohepatic circulation of $\mathrm{C}^{14}$-labeled bile salts in disorders of the distal small bowel. Gastroenterology, 55, 5-16.

Hepner, G. W., Hofmann, A. F., and Thomas, P. J. (1972). Metab- olism of steroid and amino acid moieties of conjugated bile acids in man. J. clin. Invest., 51, 1898-1905.

Hofmann, A. F. (1962). Thin-layer adsorption chromatography of free and conjugated bile acids on silicic acid. J. Lipid Res., 3, $127-128$.

Iwata, T., and Yamasaki, K. (1964). Enzymatic determination and thin-layer chromatography of bile acids in blood. J. Biochem. (Tokyo), 56, 424-431.

Jeans, W. D. (1972). An evaluation of radiological signs in small bowel examinations in children. Clin. Radiol., 23, 78-86.

Low-Beer, T. S., Heaton, K. W., Heaton, S. T., and Read, A. E. (1971). Gallbladder inertia and sluggish enterohepatic circulation of bile-salts in coeliac disease. Lancet, 1, 991-994.

Low-Beer, T. S., Pomare, E. W., and Morris, J. S. (1972). Control of bile salt synthesis. Nature [new Biol.], 238, 215-216.

Miettinen, T. A. (1968). Intestinal and faecal bile acids in malabsorption. Lancet, 2, 358.

Miettinen, T. A., and Siurala, M. (1971). Micellar solubilization of intestinal lipids and sterols in gluten enteropathy and liver cirrhosis. Scand. J. Gastroent., 6, 527-535.

Spiro, H. M. (1970) Clinical Gastroenterology. Collier-Macmillan, London. 